Claremont Colleges

Scholarship@ Claremont

All HMC Faculty Publications and Research

HMC Faculty Scholarship

$1-26-2004$

\title{
Limits to Performance Improvement Provided by Balanced Interferometers and Balanced Detection in OCT/OCM Instruments
}

David Liao

Adam E. Pivonka

Brendan R. Haberle

Daniel C. Petersen

Harvey Mudd College

Barbara M. Hoeling

California State Polytechnic University - Pomona

See next page for additional authors

\section{Recommended Citation}

David Liao, Adam E. Pivonka, Brendan R. Haberle, Daniel C. Petersen, Barbara M. Hoeling and Richard C. Haskell, "Limits to performance improvement provided by balanced interferometers and balanced detection in OCT/OCM instruments", Proc. SPIE 5316, 467 (2004); http://dx.doi.org/10.1117/12.529813

This Article is brought to you for free and open access by the HMC Faculty Scholarship at Scholarship @ Claremont. It has been accepted for inclusion in All HMC Faculty Publications and Research by an authorized administrator of Scholarship @ Claremont. For more information, please contact scholarship@cuc.claremont.edu. 


\section{Authors}

David Liao, Adam E. Pivonka, Brendan R. Haberle, Daniel C. Petersen, Barbara M. Hoeling, and Richard C. Haskell 


\title{
Limits to performance improvement provided by balanced interferometers and balanced detection in OCT/OCM instruments
}

\author{
David Liao, Adam E. Pivonka, Brendan R. Haberle, Daniel C. Petersen, \\ Barbara M. Hoeling, and Richard C. Haskell* \\ Physics Department, Harvey Mudd College, Claremont, CA 91711
}

\begin{abstract}
We compare the dynamic range of OCT/OCM instruments configured with unbalanced interferometers, e.g., Michelson interferometers, with that of instruments utilizing balanced interferometers and balanced photodetection. We define the dynamic range (DR) as the ratio of the maximum fringe amplitude achieved with a highly reflecting surface to the rootmean-square (rms) noise. Balanced systems achieve a dynamic range 2.5 times higher than that of a Michelson interferometer, enabling an image acquisition speed roughly 6 times faster. This maximum improvement occurs at light source powers of a few milliwatts. At light source powers higher than $30 \mathrm{~mW}$, the advantage in acquisition speed of balanced systems is reduced to a factor of 4 . For video-rate imaging, the increased cost and complexity of a balanced system may be outweighed by the factor of 4 to 6 enhancement in image acquisition speed. We include in our analysis the "beat-noise" resulting from incoherent light backscattered from the sample, which reduces the advantage of balanced systems. We attempt to resolve confusion surrounding the origin and magnitude of "beat-noise", first described by L. Mandel in 1962. Beat-noise is present in both balanced and unbalanced OCT/OCM instruments.
\end{abstract}

Keywords: Dynamic range, signal-to-noise ratio, optical coherence tomography, optical coherence microscopy

\section{INTRODUCTION}

The dynamic range of an OCT/OCM instrument is the critical factor in determining the trade-off between image quality and image acquisition speed. Several research groups ${ }^{1-4}$ have studied improvements in OCT dynamic range achieved with balanced interferometer configurations. For example, a Mach-Zender interferometer can be used instead of an unbalanced Michelson interferometer so that photons from the OCT broadband light source are used more efficiently. In addition, balanced detectors can reject common-mode intensity noise (Bose-Einstein photon bunching) and consequently boost the dynamic range. It is now common to employ both of these techniques in so-called "dualbalanced" configurations, especially in instruments intended for medical diagnostic procedures requiring video-rate imaging. We report here a theoretical study suggesting that dual-balanced design can achieve a factor of 4 to 6 improvement in image acquisition speed while holding image quality constant. However, this improvement factor degrades with light source powers above $30 \mathrm{~mW}$.

Recent developments in frequency-domain OCT (FD-OCT) $)^{5-7}$ promise additional boosts in dynamic range, primarily by employing large numbers of detectors operating in parallel, consequently dividing available photons so that each detector operates in the Poisson shot noise regime - without Bose-Einstein photon bunching. These systems are more complex and expensive, at least currently, so that a thorough understanding of the potential of dual-balanced design remains useful.

\section{DYNAMIC RANGE}

The dynamic range (DR) of an OCT/OCM instrument can be defined as the ratio of the fringe amplitude measured at the surface of a mirror to the rms noise at the output of the instrument under typical imaging conditions.

$$
D R=\frac{r m s \text { fringe amplitude }}{r m s \text { detector and photon noise }}=\frac{F \eta_{h} \sqrt{P_{r e f} P_{\text {coh }}}}{r m s \text { noise }}
$$


The fringe amplitude in the numerator of Eqn. (1) is proportional to the square-root of the reference power and the power coherently reflected from the mirror. $\eta_{h}$ is the heterodyne efficiency $(\sim 1.0)$ and $F$ is a signal analysis factor approximately equal to $1 .^{8}$ The rms noise in the denominator of Eqn. (1) should be representative of instrument noise during imaging in tissue. Previous work ${ }^{3,4}$ has used the square of Eqn. (1) as a measure of the dynamic range (DR) or the signal-to-noise ratio (SNR). Often the $\mathrm{DR}$ has been quoted in $\mathrm{dB}$ as $10 \log _{10}(\mathrm{DR})$ - with our definition a comparable value in $\mathrm{dB}$ is simply $20 \log _{10}(\mathrm{DR})$.

We shall evaluate the rms noise of an OCT instrument for balanced and unbalanced configurations, and then with the help of Eqn. (1), calculate the DR for each configuration. Our results are obtained for optimized OCT/OCM instruments. For Michelson interferometers, the reference beam power has been attenuated to maximize the dynamic range for a given light source power. ${ }^{8,9}$ Similarly, the cross-efficiency (splitting ratio) of the primary fiber coupler in balanced systems has been adjusted to maximize the dynamic range for a given light source power.

\subsection{Michelson interferometer}

Fig. 1 is a schematic of the most common example of an unbalanced OCT system. The variance of the noise in this system is given by

$$
\left\langle\left(\Delta P_{\text {Michelson }}\right)^{2}\right\rangle=(N E P)^{2}(B W)+(2 B W e / \Re)\left(P_{\text {ref }}+P_{\text {incoh }}\right)+\left(B W \tau_{\text {coh }}\right)\left(P_{r e f}+P_{i n c o h}\right)^{2}+\left(B W \tau_{\text {coh }}\right) 2 P_{\text {ref }} P_{\text {incoh }}
$$

The first term on the right side of Eqn. (2) is the thermal noise of the photodetector. NEP is the noise-equivalent-power of the detector $(\mathrm{W} / \sqrt{\mathrm{Hz}})$, and $\mathrm{BW}$ is the bandwidth of the analog and digital signal-processing pathway. The second term is the photon Poisson noise. $e$ is the electronic charge and $\mathfrak{K}$ is the responsivity of the detector (A/W). $P_{\text {incoh }}$ is the power returned from the sample arm that is incoherent with the reference beam, e.g., light backscattered from tissue (or the sample fiber tip) outside the coherence volume. When imaging tissue, $P_{\text {incon }}$ is much greater than $P_{c o h}$. Therefore we have neglected $P_{c o h}$ with respect to $P_{\text {incoh }}$ and $P_{\text {ref }}$ in Eqn. (2). The third term is the Bose-Einstein photon-bunching term, sometimes called relative intensity noise. $\tau_{c o h}$ is the coherence time of the OCT light source. The fourth term is the "beat noise" first described by L. Mandel in 1962. ${ }^{10}$ This last noise term arises because two partially coherent beams $\left(P_{\text {ref }}\right.$ and $P_{\text {incoh }}$ ), rather than a single thermal field, are superposed and interfere at the detector. In deriving Eqn. (2), we have assumed that the OCT light source is unpolarized.

\section{Unbalanced Configuration (Michelson)}

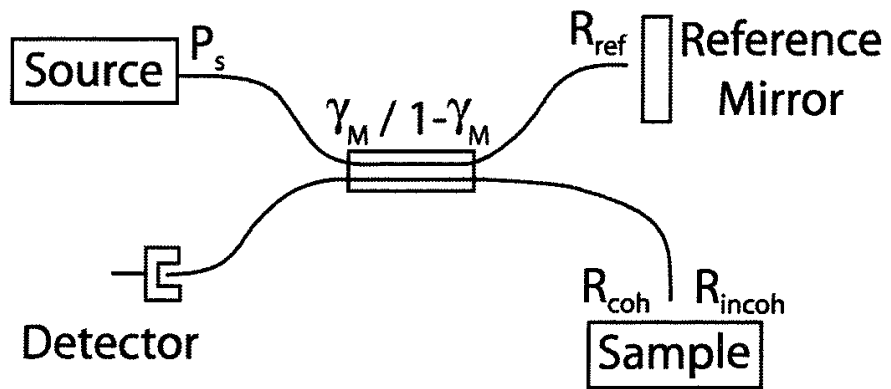

Fig. 1. Schematic of an unbalanced Michelson interferometer configuration. A single $2 \times 2$ fiber coupler is used with a cross-efficiency (splitting ratio) of $\gamma_{M}$. A single photodetector samples the interference fringes. 
The beam powers in Eqn. (2) can be written in terms of the light source power $P_{s}$ and the cross-efficiency $\gamma_{M}$ of the fiber splitter of the Michelson interferometer:

$$
P_{r e f}=P_{s} \gamma_{M}\left(1-\gamma_{M}\right) R_{r e f} \quad P_{c o h}=P_{s} \gamma_{M}\left(1-\gamma_{M}\right) R_{c o h} \quad P_{\text {incoh }}=P_{s} \gamma_{M}\left(1-\gamma_{M}\right) R_{\text {incoh }}
$$

where $R_{\text {ref }}$ is the effective reflectivity of the reference mirror (including perhaps a neutral density filter). $R_{c o h}$ is the reflectivity of the mirror surface in the coherence volume, and $R_{\text {incoh }}$ is the cumulative reflectivity of scatterers outside the coherence volume. The dynamic range of the Michelson configuration is optimized by choosing $\gamma_{M}=1 / 2$. We also reduce the value of $R_{\text {ref }}$ to maximize the DR for a given light source power. ${ }^{8,9}$

\subsection{Balanced configuration}

We examine the noise of the balanced system depicted in Fig. 2. It is similar to a balanced system denoted as "Ai" by Rollins and Izatt, ${ }^{3}$ but our balanced system employs two optical circulators for conceptual simplicity. The design of Fig. 2 utilizes a balanced interferometer and balanced detection. The noise variance of this system can be expressed as

$$
\left\langle\left(\Delta P_{\text {bul }}\right)^{2}\right\rangle=\left(N E P_{\text {dual }}\right)^{2}(B W)+(2 B W e / \Re)\left(P_{\text {ref }}+P_{\text {incoh }}\right)+\left(B W \tau_{\text {coh }}\right) 2 P_{\text {ref }} P_{\text {incoh }}
$$

In Eqn. (4), $P_{\text {ref }}$ and $P_{\text {incoh }}$ are the sum of the reference and incoherent powers, respectively, incident upon the two detectors. $N E P_{\text {dual }}$ is $\sqrt{2}$ times $N E P$ in Eqn. (2). The Bose-Einstein bunching term in Eqn. (2) has been eliminated by the balanced detection circuitry included in Fig. 2, but the beat noise term survives. ${ }^{1}$

\section{Dual-Balanced Configuration}

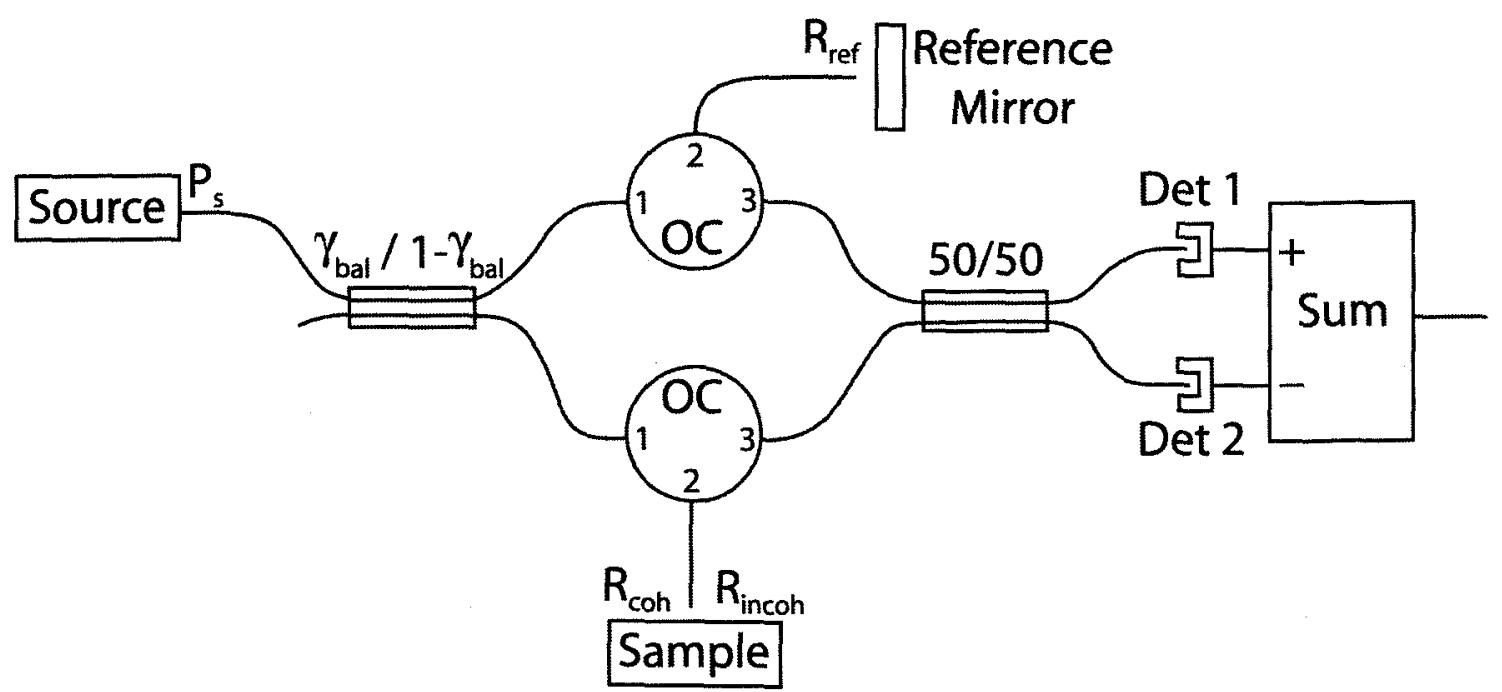

Fig. 2. Schematic of a dual-balanced OCT configuration. Two $2 \times 2$ fiber couplers are used; the primary coupler has a cross-efficiency of $\gamma_{b a l}$ while the second coupler sends 50\% to each detector. Two optical circulators (OC) are employed, one in the sample arm and one in the reference arm. Typically light is coupled from port 1 to port 2 or from port 2 to port 3 with about $85 \%$ efficiency. The outputs of two balanced detectors are subtracted to reject common-mode intensity noise. 
The beam powers in Eqn. (4) can be written in terms of the source power $P_{s}$ and the cross-efficiency $\gamma_{b a l}$ of the fiber splitter of the balanced configuration:

$$
P_{r e f}=P_{s} \gamma_{b a l} T_{c i r c}^{2} R_{r e f} \quad P_{c o h}=P_{s}\left(1-\gamma_{b a l}\right) T_{c i r c}^{2} R_{c o h} \quad P_{\text {incoh }}=P_{s}\left(1-\gamma_{b a l}\right) T_{\text {circ }}^{2} R_{\text {incoh }}
$$

Eqn. (5) is analogous to Eqn. (3) for the Michelson configuration. In Eqn. (5), $T_{\text {circ }}$ is the coupling efficiency $(\sim 0.85)$ between successive ports of an optical circulator.

The advantage of the balanced configuration is that the coupling efficiency of the fiber splitter can be varied, directing more light to the sample while keeping the reference reflectivity at its maximum value, thus maximizing the dynamic range. ${ }^{2,3,4}$

\section{RESULTS}

We have calculated the dynamic range (DR) of the Michelson and balanced configurations by inserting the square-root of Eqns. (2) and (4), respectively, into the denominator of Eqn. (1). The results for the DR are plotted in Fig. 3 as a function of light source power $P_{s}$. The values for the DR for the Michelson have been optimized with respect to $R_{r e f}$, and the values for the balanced configuration of Fig. 2 have been optimized with respect to the cross-efficiency $\gamma_{b a l}$ of the primary fiber coupler. The values of parameters used in these calculations are listed in Table 1.

\begin{tabular}{|l|l|l|l|}
\hline$F=1.175$ & $\eta_{h}=1.0$ & $N E P=1.4 \mathrm{pW} / \mathrm{Hz}^{1 / 2}$ & $B W=30 \mathrm{KHz}$ \\
\hline $\mathfrak{R}=0.95 \mathrm{~A} / \mathrm{W}$ & $\tau_{\text {coh }}=76 \mathrm{fs}$ & $R_{\text {coh }}($ mirror $)=1.0$ & $R_{\text {incoh }}=0$ or $3.5 \times 10^{-4}$ \\
\hline$\gamma_{M}=1 / 2$ & $T_{\text {circ }}=0.85$ & $N E P_{\text {dual }}=2.0 \mathrm{pW} / \mathrm{Hz}^{1 / 2}$ & \\
\hline
\end{tabular}

Table 1. Values for parameters used in Eqns. (1) through (5) in calculating data for Figs. 3 and 4.
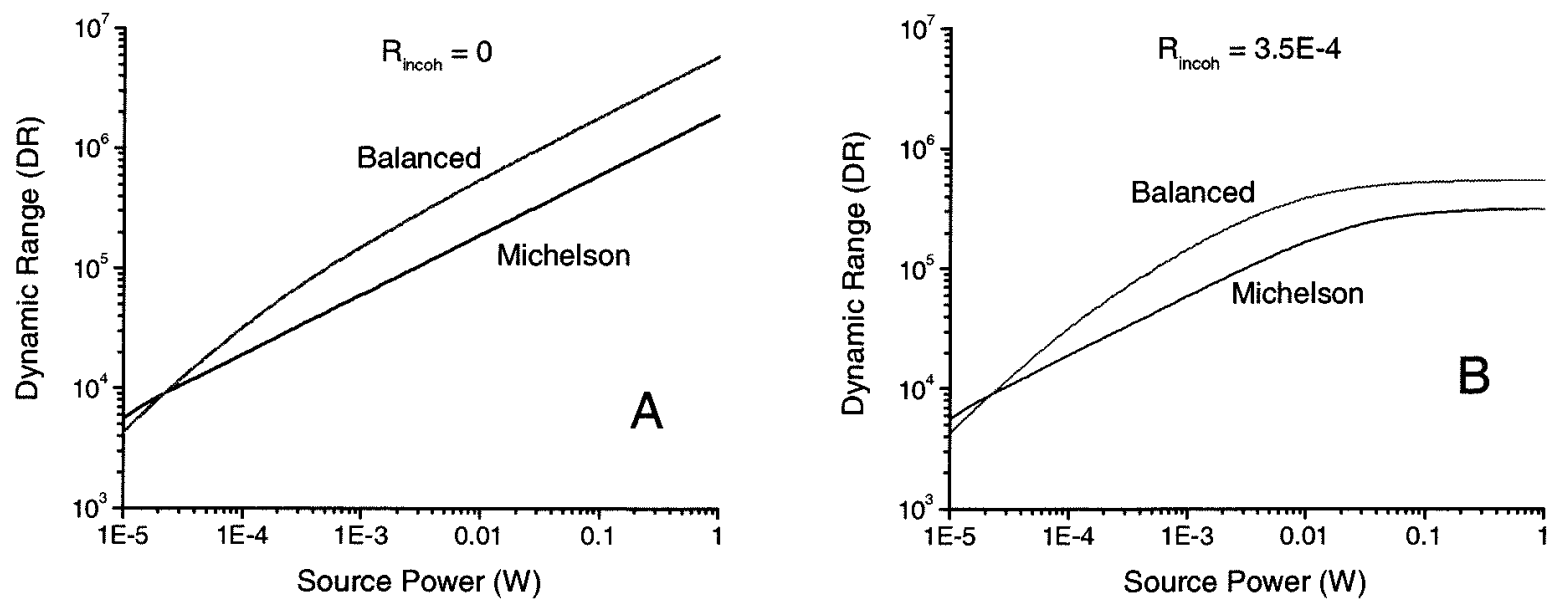

Fig. 3. Dynamic range (DR) plotted as a function of source power. (A) The incoherent reflectivity is neglected. (B) The incoherent reflectivity is taken to be $3.5 \times 10^{-4}$, a typical value for tissue. 
In Fig. 3A, the power backscattered by tissue outside the coherence volume is neglected $\left(R_{\text {incoh }}=0\right)$. Notice that the DR for both designs rises without limit, and that the asymptotic slopes are equal. This results in an asymptotic improvement factor of about 3.1 for the balanced configuration over the Michelson interferometer. In Fig. 3B, $R_{\text {incoh }}$ is assigned a typical value $\left(3.5 \times 10^{-4}\right)$ realized when imaging tissue. The resulting beat noise leads to a leveling off of the DR for both balanced and unbalanced configurations.

The ratio of the DR for the balanced configuration to that for the Michelson interferometer is plotted in Fig. 4 as a function of source power. Notice that the maximum advantage of the balanced system is about 2.5 and occurs at $3 \mathrm{~mW}$ of source power. At a source power of $30 \mathrm{~mW}$, the advantage of the balanced system has fallen to a factor of 2.0 .

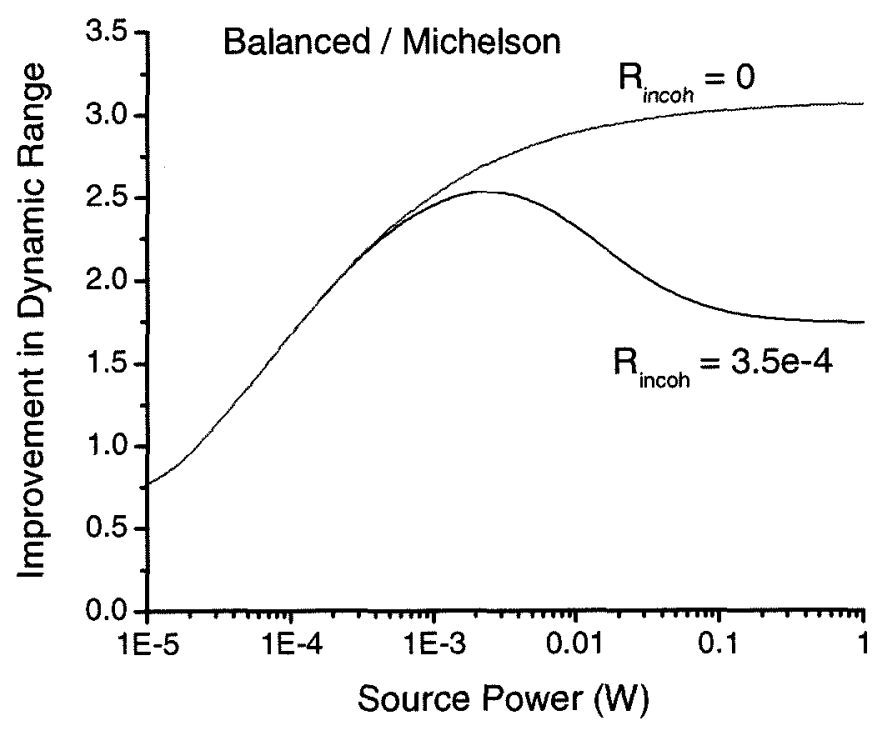

Fig. 4. The improvement in the DR of the balanced design over that of the Michelson configuration is plotted as a function of source power. For realistic values of the incoherent backscattering appropriate to imaging of tissue, the advantage of the balanced system peaks at 2.5 for approximately $3 \mathrm{~mW}$ of source power.

\section{CONCLUSIONS}

The phenomenon of "beat noise" was first discussed in connection with the Alford and Gold effect in $1962 .{ }^{10} \mathrm{~L}$. Mandel described it as an intensity correlation effect. Indeed, the mathematics describing beat noise is very similar to that for Bose-Einstein photon bunching, and the approximate expression for beat noise in Eqn. (2) (last term) is the same as the cross term in the photon bunching expression (third term in Eqn. (2)).

The most striking effect of beat noise in OCT is the upper limit placed on the dynamic range at high source powers. The curves in Fig. 3B begin to bend over noticeably at source powers as low as $10 \mathrm{~mW}$. In contrast, the improvement factor in DR for balanced systems over Michelson interferometers is affected only modestly by beat noise. For example, the DR improvement ratio is roughly 2.7 without beat noise at $3 \mathrm{~mW}$ of source power, and is reduced slightly to 2.5 in the presence of beat noise.

It is clear that a dual-balanced OCT configuration can provide a factor of 4 to 6 increase in image acquisition speed. Balanced detectors and optical circulators add complexity and expense to the instrument, but the power of video-rate imaging may well justify the extra effort. 


\section{ACKNOWLEDGMENTS}

We gratefully acknowledge support for this work from the National Science Foundation Grant DBI-0137973.

\section{REFERENCES}

1. K. Takada, "Noise in optical low-coherence reflectometry," IEEE J. Quantum. Electron. 34, 1098-1108 (1998).

2. B. E. Bouma and G. J. Tearney, "Power-efficient nonreciprocal interferometer and linear-scanning fiber-optic catheter for optical coherence tomography," Optics Lett. 24(8) 531-533 (1999).

3. A. Rollins and J. Izatt, "Optimal interferometer designs for optical coherence tomography," Optics Lett. 24, 14841486 (1999).

4. A. Gh. Podoleanu, "Unbalanced versus balanced operation in an optical coherence tomography system," Appl. Optics 39, 173-182(2000).

5. R. Leitgeb, C. K. Hitzenberger, and A. F. Fercher, "Performance of fourier domain vs. time domain optical coherence tomography," Optics Express 11(8) 889-894 (2003).

6. M. A. Choma, M. V. Sarunic, C. Yang, and J. A. Izatt, "Sensitivity advantage of swept source and Fourier domain optical coherence tomography," Optics Express 11(18) 2183-2189 (2003).

7. J. F. de Boer, B. Cense, B. H. Park, M. C. Pierce, G. J. Tearney, and B. E. Bouma, "Improved signal-to-noise ratio in spectral-domain compared with time-domain optical coherence tomography," Optics Lett. 28(21) 2067-2069.

8. B. M. Hoeling, A. D. Fernandez, R. C. Haskell, E. Huang, W. R. Myers, D. C. Petersen, S. E. Ungersma, R. Wang, M. E. Williams, and S. E. Fraser, "An optical coherence microscope for 3-dimensional imaging in developmental biology," Optics Express 6(7) 136-146 (2000).

9. W. V. Sorin and D. M. Baney, "A simple intensity noise reduction technique for optical low-coherence reflectometry," IEEE Photonics Tech. Lett. 4, 1404-1406 (1992).

10. L. Mandel, "Interference and the Alford and Gold effect," J. Opt. Soc. Am. 52, 1335-1340 (1962).

*Richard Haskell@hmc.edu; phone 909-607-2768; fax 909-621-8887 